\title{
Dynamic measurements on ordered lattices
}

\author{
B. Van der Vorst*, D. Van den Ende, J. Mellema \\ Rheology Group, Faculty of Applied Physics, University of Twente, P.O. Box 217. 7500AE Enschede. The Netherlands
}

\section{Introduction}

Suspensions of polystyrene spheres crystallise due to strongly repulsive interactions between the spheres. These liquid crystals are expected to behave solid-like reflected by a static modulus and a yield stress. Many investigators measured the dynamic moduli in a small frequency range $[1,2]$. They compare the experimental dynamic storage modulus with a theoretical static modulus assuming them to be identical. Both experiments and theory do not investigate the behaviour of the dynamic moduli.

In the present study the dynamic moduli are measured over a wide range of frequencies $(\omega / 2 \pi \in[0.001-10 \mathrm{~Hz}])$ with a home-made apparatus. The volume fractions of the latex crystals ranged from 0.015 to 0.30 . We expect an order-disorder transition to take place in this range and a change in the dynamic moduli.

\section{Experimental set-up}

On the basis of a recipe described by Goodwin et al. [3] our fluids are prepared by a polymerisation reaction in water of styrene and potassium sulphonate initiator. This reaction produces negative-charged polystyrene spheres. The size distribution of the resulting spherical latex particles is very monodisperse with a radius of $250 \mathrm{~nm}$. The negative surface charge is caused by potassium sulfonate

\footnotetext{
* Corresponding author.
}

end groups which dissolve in water. The sulphonate groups are anchored to the particle surface while the potassium counterions are dissolved in the water. The remnants of the polymerisation reaction are washed away by ultrafiltration. With an ion-exchange resin all excessive electrolyte is extracted from the latex and the potassium counterions are exchanged for hydrogen ions. After this purification the number of counterions ( = number of sulphonate groups) is determined by conductometric titration of the latex with sodium hydroxide. The surface charge density is then calculated from this number, the particle radius and the volume fraction. Mass fractions were determined by drying approximately $1 \mathrm{~g}$ of latex at $50^{\circ} \mathrm{C}$. The volume fraction was calculated from the dry weight with a particle density of $1.05 \mathrm{~g} / \mathrm{ml}$.

The deionisation also maximises the electrostatic repulsion between the particles. The total interaction energy consists of van der waals attraction, hard-sphere repulsion and electrostatic repulsion and is for low electrolyte concentrations dominated by the latter one. Because the particles are locked up in a closed volume the electrostatic repulsion forces the particles to order in a crystal structure (BCC or FCC).

The dynamic experiments are performed with a home-made instrument [4]. In the apparatus the latex is confined between two concentric cylinders. The inner cylinder is oscillating in a forced harmonic vibration driven via a torsion spring by the motor. The dynamic moduli are determined by measuring the amplitude ratio $R$ and the phase difference $\varphi$ between the cylinder and the 
motor,

$G^{\prime}=\frac{K}{U}(R \cos \varphi-1)+\frac{I}{U}\left(\omega^{2}\right.$,

$G^{\prime \prime}=\frac{K}{U} R \sin \varphi-\frac{D}{U} \omega$.

Here $K$ is the torsion constant of the spring, $I$ is the moment of inertia of the inner cylinder, $D$ is the damping of the air bearing and $U$ is a geometrical factor which are determined by a calibration procedure.

For the experiments shown here the strain amplitudes are smaller than 0.005 which ensures linear experiments in these cases.

\section{Theory}

Here we will focus on the volume fraction dependency of the static shear modulus $\left(G_{0}\right)$ as determined from Fig. 2.

Several researchers have studied the static modulus of an ordered suspension theoretically [5-8]. Following Buscall et al. [7] the stress in the crystal is calculated from

$\overrightarrow{\mathrm{T}}=-\frac{1}{V} \sum_{i=1}^{N_{\text {tot }}} \sum_{\substack{j=1 \\ j \neq i}}^{N_{\text {tot }}} \boldsymbol{F}_{i j} \boldsymbol{r}_{i}$.

Here $V$ is the total volume of the crystal occupied by the $N_{\text {tot }}$ particles, $F_{i j}$ is the electrostatic force on particle $i$ due to its $j$ th neighbour and $\boldsymbol{r}_{i}$ is the position of particle $i$.

To derive an expression for the static modulus a small strain $\gamma$ is applied to the crystal (here we consider a deformation of the crystal in the $x$-direction with the gradient of the strain in the $z$-direction). Then, after making a Taylor expansion of Eq. (3) with respect to the displacements caused by the strain, we study the $x z$-component of the stress since we are interested in the shear modulus of the crystal. By averaging $\overrightarrow{\mathrm{T}}_{x x}$ over all possible crystal orientations the polycrystallinity is taken into account and eventually leads to [9]

$$
\left\langle\overrightarrow{\mathrm{T}}_{x z}\right\rangle=G_{0} \gamma=\frac{\phi_{m} N}{5 \pi R}\left(\psi^{\prime \prime}+4 \psi^{\prime} R^{-1}\right) \gamma
$$

Here $\psi$ is the pair interaction energy between two particles with centres at a distance $R$ from each other, $\phi_{m}$ is the volume fraction at close packing for the crystal structure considered and $N$ is the number of nearest neighbours for the crystal structure considered. In this derivation the influence of the particles at the sample boundary is omitted and only the nearest neighbour forces are taken into account. These assumptions are validated by the strong screening of the interaction energy between the particles. For the pair interaction energy we use the result derived by Bell et al. [10], who gave the expression for the interaction energy between a pair of isolated particles placed at a distance $R$ from each other,

$\psi(R)=4 \pi \varepsilon_{\mathrm{r}} \varepsilon_{0} \Phi^{2} \frac{a^{2}}{R} \mathrm{e}^{-\kappa(R-2 a)}$.

Here $\kappa$ is the reciprocal of the Debije screening length, $\Phi$ is the surface potential on the particles and $\varepsilon_{\mathrm{r}} \varepsilon_{0}$ is the dielectric constant of the fluid. Since expression (5) is derived for an isolated pair of particles with low surface potentials it is not directly clear that it can be applied to describe the interaction energy between two particles with a surface potential in a crystal. To elucidate this we first mention that the pair interaction energy is fully determined by the behaviour of the electric potential at the midplane between the two particles $[10-12]$. If the two particles are separated sufficiently the electric potential and its gradient at the midplane can be approximated by a linear superposition of two single-particle contributions which have the form

$\varphi(r)=\Phi_{\mathrm{a}} \frac{a}{r} \mathrm{e}^{-\kappa_{\mathrm{e}}(r-a)}$.

Here $r$ is the distance to centre of the particle, $a$ is the particle radius, $\Phi_{\mathrm{a}}$ is an apparent surface potential of the particle and $\kappa_{\mathrm{e}}$ is the effective screening parameter. From the analytic approximation for the electric potential at the midplane Bell et al. [10] derived equation (5) for the pair interaction energy.

In the crystal the electric potential "far away" from the particles is conceived as a summation over many single-particle contributions of the form (6). In order to obtain values for the apparent potential $\Phi_{\mathrm{a}}$ and the effective screening parameter $\kappa_{\mathrm{e}}$ in the 
crystal the electric potential is solved numerically by integration of the Poisson-Boltzmann (PB) equation. Because of the translation symmetry of the crystal the electric potential only has to be solved in one Wigner-Scitz cell. A commonly used technique to simplify the calculation is to replace polyhedron cell by a spherical cell of radius $h$. The $\mathrm{PB}$-equation which has to be solved numerically is

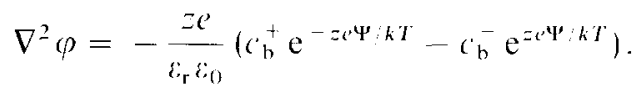

Here $\mathrm{c}_{\mathrm{b}}^{+}$and $\mathrm{C}_{\mathrm{b}}^{-}$are the spatial concentrations of the positive and negative ions at the cell boundary, $z$ is the valence of the counterions and of the $z z$ electrolyte, $c$ is the electron charge, $k$ is the Boltzmann factor and $T$ is the temperature.

The boundary condition on the particle surface is

$\left.\frac{\mathrm{d} \varphi}{\mathrm{d} r}\right|_{r=a}=-\frac{\sigma}{i_{\mathrm{r}} \delta_{0}}$.

Demanding that the cell is electrical neutral results in the boundary condition on the surface

$\left.\frac{\mathrm{d} \varphi}{\mathrm{d} r}\right|_{r=h}=0$.

We integrate the PB-equation with an integration scheme based on the one used by Alexander et al. [13]. The result of this calculation is shown in Fig. 1 where the solid line represents the calculated electric PB-potential in the cell.

Since the PB-potential becomes small near the cell boundary the PB-equation can be linearised to the Debije Hückel (DH) equation. Consequently, near the cell surface, the numerical PB-potential can be approximated by an analytic DH-potential which is shown as a dashed line in Fig. 1. From this DH-potential the apparent surface potential $\Phi_{a}$ and the effective screening parameter $\kappa_{c}$ are determined.

Substitution of Eq. (5) in Eq. (4) and interpreting the screening parameter $k$ as an effective screening parameter $\kappa_{\mathrm{e}}$ and the surface potential $\Phi$ as an apparent surface potential $\Phi_{i t}$ the static shear modulus becomes

$G_{0}=4 \pi x \varepsilon_{\mathrm{r}} \xi_{0} a^{2} \Phi_{\mathrm{a}}^{2} \frac{\left(\kappa_{\mathrm{c}}^{2} R^{2}-2 \kappa_{\mathrm{c}} R-2\right)}{R^{4}} \mathrm{e}^{-\kappa_{\mathrm{c}}(R-2 a)}$

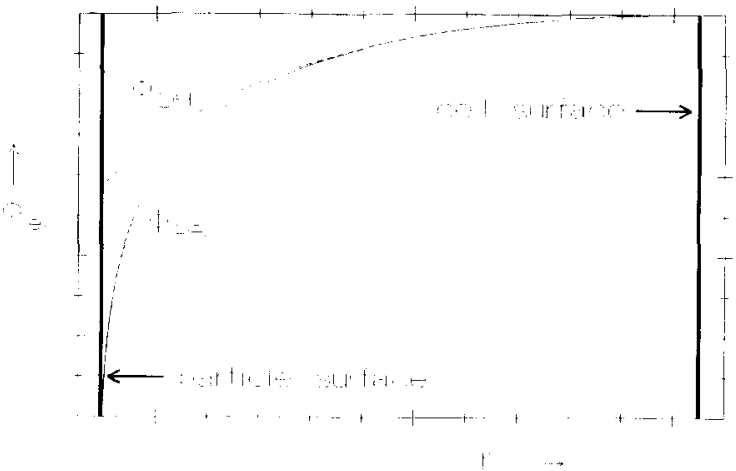

Fig. 1. The electric potential. within the cell, as a function of the distance. The numerical solution of the PB-equation (solid line) is near the coll boundary approximated by the analytical solution of the DH-cepuation (dashed line).

with

$\chi=N \phi_{\mathrm{m} /} / 5 \pi$

\section{Results}

The dynamic moduli have been measured as a function of the frequency for eight different volume fractions. The result for the storage modulus is shown in Fig. 2.

From Fig. 2 it can be seen that for high volume fraction $(\phi>0.10)$ the storage modulus becomes nearly constant for all frequencies. In literature this storage modulus is assumed to be equal to the static shear modulus of the liquid crystal due to the electrostatic forces.

The only unknown in our experiments was the excess electrolyte concentration. By adjusting this concentration we fitted the static shear modulus (10) to the data. A satisfying fit has been achieved for an excess electrolyte concentration of $4.7 \times 10^{-6} \mathrm{M}$ and is shown in Fig. 3. This excess electrolyte concentration is reasonable for a latex that is purified with an ion-exchange resin. In Fig. 3 also data on the static shear modulus measured by Chow and Zukoski [14] are shown. Here all parameters were known so no free parameters were left to adjust the model to the data.

Next the theoretical static modulus is compared with the experimental moduli determined by other 


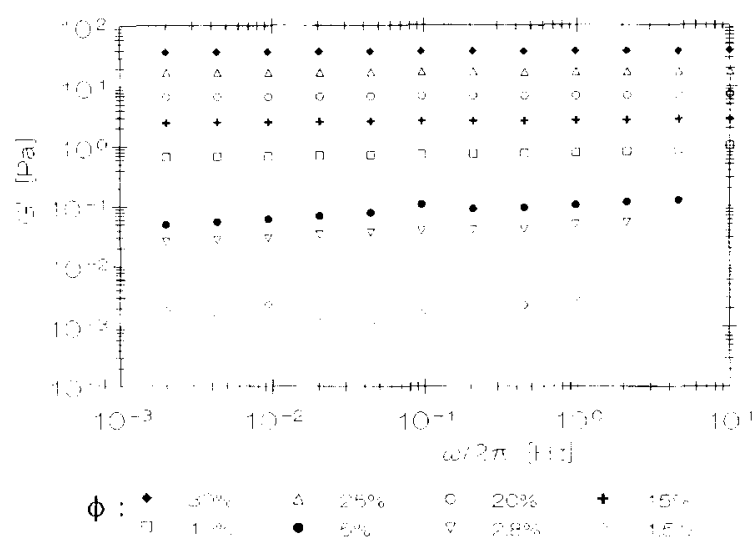

Fig. 2. Storage modulus of a crystallised latex measured for a wide range of frequencies for eight volume fractions.

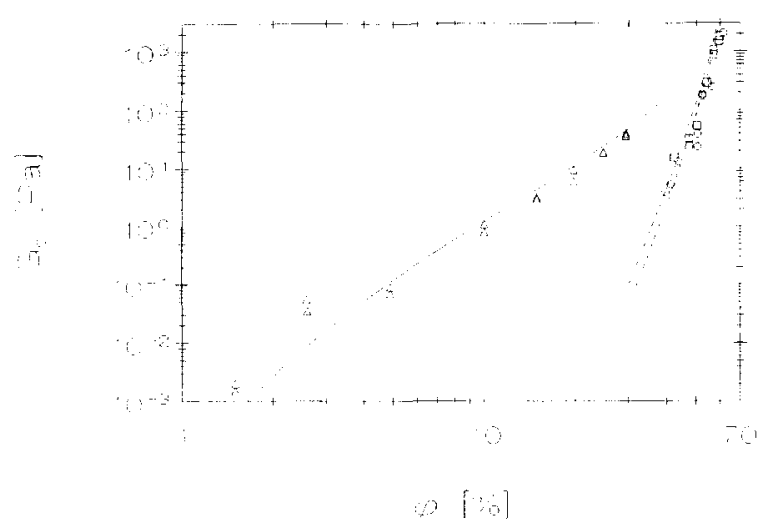

Fig. 3. The static shear modulus as a function of the volume fraction. Data $(\Delta)$ from Fig. 2 and data $(\square, O)$ from Chow and Zukoski [14]. Solid and dashed lines are theoretical calculations.

researchers for different particle radii, volume fractions, surface charge densities and excess electrolyte concentrations. To investigate the capabilities of our model the static shear moduli measured by several authors are scaled on a universal curve. Rewriting Eq. (10) leads to the scaling equation

$\frac{G_{0} a^{2}}{C \Phi_{\mathrm{i}}^{2}}=F\left(\kappa_{\mathrm{e}} a, \phi\right)$

with

$C=4 \pi x \ddot{r}_{\mathrm{r}} \dot{ }_{0}$

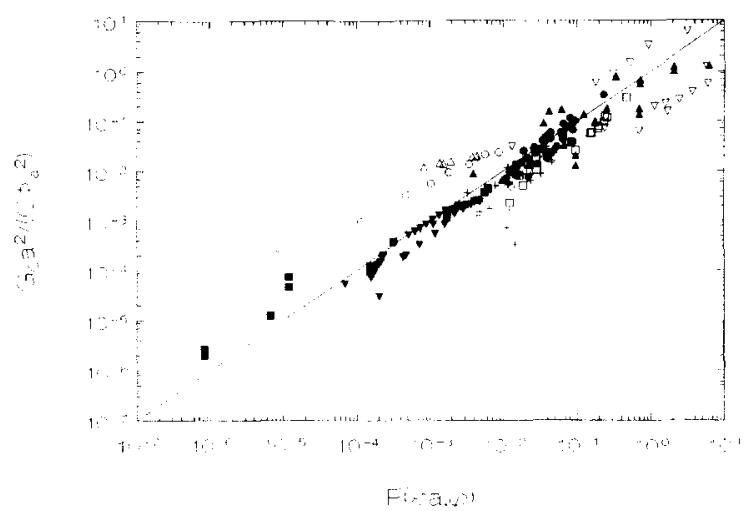

Fig. 4. The sealed modulus plotted versus $F\left(\kappa_{0} a, \phi\right)$ (see Eq. (11). Data (n) measured by us, the other authors cited in this figure can be found in Van der Vorst et al. [16].

and

$$
\begin{aligned}
& F\left(\kappa_{\mathrm{c}} a, \phi\right)= \\
& {\left[\left(\kappa_{\mathrm{c}} R\right)^{2}-2 \kappa_{\mathrm{c}} R-2\right]\left(\frac{\kappa_{\mathrm{c}} a}{\kappa_{\mathrm{c}} R}\right)^{4} \mathrm{e}^{-\kappa_{\mathrm{e}}(k \cdots 2 a !} .}
\end{aligned}
$$

The result of this scaling is shown in Fig. 4.

\section{Conclusions}

The static shear modulus measured by several authors under various experimental conditions is reasonably well described by the simple model proposed here. Deviations may stem from the fact that we used a spherical cell instead of the Wigner-Seitz cell which implies that the static potential surrounding a particle is also spherical symmetric. However, we believe that the main physical aspects are incorporated quantitatively in this model. Furthermore, the pair interaction energy derived for a pair of isolated charged spheres with a low surface potential is applied successfully to a many particle system consisting of particles with a high surface potential. Hereto we interpreted the surface potential $\Phi$ and the screening parameter $k$ appearing in the expression for the interaction energy as an apparent surface potential $\phi_{\text {: }}$ and an effective screening parameter $\kappa_{c}$. 


\section{References}

[1] D.W. Benzing and W.B. Russel, J. Colloid Interface Sei. 83 (1981) 178-190.

[2] S. Mitaku, T. Ohtsuki and K. Okano, Jpn. J. Appl. Phys. $19(1980) 439-448$.

[3] J.W. Goodwin. J. Hearn, C.C. Ho and R.H. Ottewill, Br. Poly. J. 5 (1973) $347-362$.

[4] H.J.E. Smits, D. van den Ende, G.J. Beukema. E.G. Altena and P.H.J. Spitteler, Rheol Acta 31 (1992) 471480.

[5] Ohtsuki et al. (1978).

[6] Ohtsuki et al. (1981).

[7] Buscall et al. (1982).

[8] W.B. Russel and D.W. Benzing, J. Colloid Interface Sci. 83 (1981) $163 \cdots 177$.
[9] N.J. Wagner. J. Colloid Interface Sci. 161 (1993) $169-181$.

[10] G.M. Bell. S. Levine and L.N. McCartncy, J. Colloid Interface Sci. 33, (1970) 335359

[11] Glendinning and Russel (1983)

[12] S.L. Carnie, D.Y.C. Chan and J.S. Gunning, Langmuir 10) (1994) $2993 \cdots 3009$.

[1.3] S. Alexander. P.M. Chaikin. P. Grant. G.J. Morales. P. Pincus and D. Hone. J. Chem. Phys. 80, (1984) 5776-5781.

[14] Chow and Zukoski (1995)

[15] L.B. Chen and C.F. Zukoski. J. Chem. Soc. Faraday Trans. $86(1990) 2629 \cdot 26.39$.

[16] B. Van der Vorst, D. Van den Ende and J. Mellema. J. Rheol. 39 (1995) 1 183 1200 . 